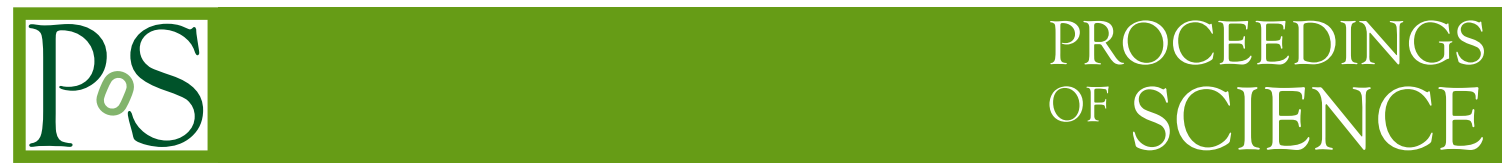

\title{
Transverse Force on Quarks in DIS
}

Matthias Burkardt ${ }^{* \dagger}$

New Mexico State University

E-mail: burkardt@nmsu.edu

Generalized Parton Distributions (GPDs) provide information on the distribution of quarks in impact paarmeter space. For transversely polarized nucleons, these impact parameter distributions are transversely distorted and this deviation from axial symmetry leads on average to a net transverse force from the spectators on the active quark in a DIS experiment. This force when acting along the whole trajectory of the active quark leads to a transverse single-spin asymmetries. For a longitudinally polarized nucleon target, the transverse force implies a torque acting on the quark Orbital Angular Momentum (OAM). The resulting change in OAM as the quark leaves the target equals the difference between the Jaffe-Manohar and Ji OAMs.

XXIII International Workshop on Deep-Inelastic Scattering

27 April - May 12015

Dallas, Texas

\footnotetext{
${ }^{*}$ Speaker.

${ }^{\dagger}$ This work was supported by the DOE under grant number DE-FG03-95ER40965.
} 


\section{Angular Momentum Decompositions}

Since the famous EMC experiments revealed that only a small fraction of the nucleon spin is due to quark spins[1], there has been a great interest in 'solving the spin puzzle', i.e. in decomposing the nucleon spin into contributions from quark/gluon spin and orbital degrees of freedom. In this effort, the Ji decomposition[2]

$$
\frac{1}{2}=\frac{1}{2} \sum_{q} \Delta q+\sum_{q} L_{q}^{z}+J_{g}^{z}
$$

appears to be very useful: through GPDs, not only the quark spin contributions $\Delta q$ but also the quark total angular momenta $J_{q} \equiv \frac{1}{2} \Delta q+L_{q}^{z}$ (and by subtracting the spin piece also the the quark orbital angular momenta $L_{q}^{z}$ ) entering this decomposition can be accessed experimentally. In the Ji decomposition (1.1) the quark OAM is defined as the expectation value

$$
L_{q}^{z}=\int d^{3} r\left\langle P S\left|q^{\dagger}\left(\vec{r} \times \frac{1}{i} \vec{D}\right)^{z} q\right| P S\right\rangle /\langle P S \mid P S\rangle
$$

in a nucleon state polarized in the $+\hat{z}$ direction. Here $\vec{D}=\vec{\partial}-i g \vec{A}$ is the gauge-covariant derivative. The main advantages of this decomposition are that each term can be expressed as the expectation value of a manifestly gauge invariant local operator and that the quark total angular momentum $J^{q}=\frac{1}{2} \Delta q+L^{q}$ can be related to GPDs[2] and is thus accessible in deeply virtual Compton scattering and deeply virtual meson production and can also be calculated in lattice gauge theory.

Jaffe and Manohar have proposed an alternative decomposition of the nucleon spin, which does have a partonic interpretation, and in which also two terms, $\frac{1}{2} \Delta q$ and $\Delta G$, are experimentally accessible [3]

$$
\frac{1}{2}=\frac{1}{2} \sum_{q} \Delta q+\sum_{q} \mathscr{L}^{q}+\Delta G+\mathscr{L}^{g}
$$

In this decomposition the quark OAM is defined as

$$
\mathscr{L}^{q} \equiv \int d^{3} r\left\langle P S\left|q_{+}^{\dagger}\left(\vec{r} \times \frac{1}{i} \vec{\partial}\right)^{z} q_{+}\right| P S\right\rangle /\langle P S \mid P S\rangle,
$$

where light-cone gauge $A^{+}=0$ is implied. Although Eq. (1.4) is not manifestly gauge invariant as written, gauge invariant extensions can be defined $[4,5]$. Indeed, manifestly gauge invariant definitions exist for each of the terms in Eq. (1.3) which with the exception of $\Delta q$ involve matrix elements of nonlocal operators. In light-cone gauge those nonlocal operators reduce to a local operator, such as Eq. (1.4).

\section{TMDs and OAM from Wigner Distributions}

Wigner distributions can be defined as off forward matrix elements of non-local correlation functions $[6,7,8]$ with $P^{+}=P^{+\prime}, P_{\perp}=-P_{\perp}^{\prime}=\frac{q_{\perp}}{2}$

$$
W^{\mathscr{U}}\left(x, \vec{b}_{\perp}, \vec{k}_{\perp}\right) \equiv \int \frac{d^{2} \vec{q}_{\perp}}{(2 \pi)^{2}} \int \frac{d^{2} \xi_{\perp} d \xi^{-}}{(2 \pi)^{3}} e^{-i \vec{q}_{\perp} \vec{b}_{\perp}} e^{i\left(x P^{+} \xi^{-}-\vec{k}_{\perp} \vec{\xi}_{\perp}\right)}\left\langle P^{\prime} S^{\prime}\left|\bar{q}(0) \Gamma \mathscr{U}_{0 \xi} q(\xi)\right| P S\right\rangle .
$$


Throughout this paper, we will chose $\vec{S}=\vec{S}^{\prime}=\hat{\vec{z}}$. Furthermore, we will focus on the 'good' component by selecting $\Gamma=\gamma^{+}$. To ensure manifest gauge invariance, a Wilson line gauge link $\mathscr{U}_{0 \xi}$ connecting the quark field operators at position 0 and $\xi$ is included. The issue of choice of path for the Wilson line will be addressed below.

In terms of Wigner distributions, TMDs and OAM can be defined as [4]

$$
\begin{aligned}
f\left(x, \vec{k}_{\perp}\right) & =\int d x d^{2} \vec{b}_{\perp} d^{2} \vec{k}_{\perp} \vec{k}_{\perp} W^{\mathscr{U}}\left(x, \vec{b}_{\perp}, \vec{k}_{\perp}\right) \\
L_{\mathscr{U}} & =\int d x d^{2} \vec{b}_{\perp} d^{2} \vec{k}_{\perp}\left(\vec{b}_{\perp} \times \vec{k}_{\perp}\right)^{z} W^{\mathscr{U}}\left(x, \vec{b}_{\perp}, \vec{k}_{\perp}\right) .
\end{aligned}
$$

No issues with the Heisenberg uncertainty principle arise here since only perpendicular combinations of position $\vec{b}_{\perp}$ and momentum $\vec{k}_{\perp}$ are needed simultaneously in order to evaluate the integral for $L_{\mathscr{U}}$.

A straight line connecting 0 and $\xi$ for the Wilson line in $\mathscr{U}_{0 \xi}$ results in [7]

$$
L_{\text {straight }}^{q}=L_{J i}^{q} \text {. }
$$

However, depending on the context, other choices for the path in the Wilson link $\mathscr{U}$ should be made. Indeed for TMDs probed in SIDIS the path should be taken to be a straight line to $x^{-}=\infty$ along (or, for regularization purposes, very close to) the light-cone. This particular choice ensures proper inclusion of the FSI experienced by the struck quark as it leaves the nucleon along a nearly light-like trajectory in the Bjorken limit. However, a Wilson line to $\xi^{-}=\infty$, for fixed $\vec{\xi}_{\perp}$ is not yet sufficient to render Wigner distributions manifestly gauge invariant, but a link at $\xi^{-}=\infty$ must be included to ensure manifest gauge invariance. While the latter may be unimportant in some gauges, it is crucial in light-cone gauge for the description of TMDs relevant for SIDIS [9].

Let $\mathscr{U}_{0 \xi}^{+L C}$ be the Wilson path ordered exponential obtained by first taking a Wilson line from $\left(0^{-}, \overrightarrow{0}_{\perp}\right)$ to $\left(\infty, \overrightarrow{0}_{\perp}\right)$, then to $\left(\infty, \vec{\xi}_{\perp}\right)$, and then to $\left(\xi^{-}, \vec{\xi}_{\perp}\right)$, with each segment being a straight line (Fig. 1) [5]. The shape of the segment at $\infty$ is irrelevant as the gauge field is pure gauge there, but it
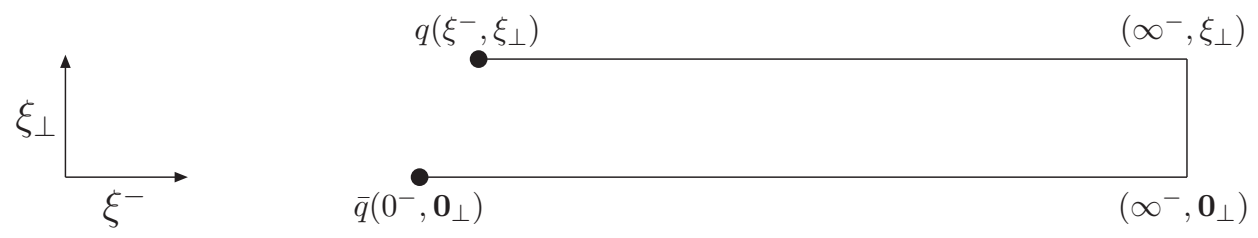

Figure 1: Illustration of the path for the Wilson line gauge link $\mathscr{U}_{0 \xi}^{+L C}$ entering $W^{+L C}$

is still necessary to include a connection at $\infty$ and for simplicity we pick a straight line. Likewise, with a similar 'staple' to $-\infty$ we define the Wilson path ordered exponential $\mathscr{U}_{0 \xi}^{-L C}$, and using those light-like gauge links ${ }^{1}$, we define

$$
W^{ \pm L C}\left(x, \vec{b}_{\perp}, \vec{k}_{\perp}\right) \equiv \int \frac{d^{2} \vec{q}_{\perp}}{(2 \pi)^{2}} \int \frac{d^{2} \xi_{\perp} d \xi^{-}}{(2 \pi)^{3}} e^{-i \vec{q}_{\perp} \cdot \vec{b}_{\perp}} e^{i\left(x P^{+} \xi^{-}-\vec{k}_{\perp} \vec{\xi}_{\perp}\right)}\left\langle P^{\prime} S^{\prime}\left|\bar{q}(0) \Gamma \mathscr{U}_{0 \xi}^{ \pm L C} q(\xi)\right| P S\right\rangle .
$$

This definition for $W^{+L C}$ the same as that in Ref. [5] and similar to that of $W_{L C}$ in Ref. [7] (the link segment at $\xi^{-}=\infty$ was not included in the definition of $W_{L C}$ ).

\footnotetext{
${ }^{1}$ Subtleties in regularizing/renormalizing such objects are addressed in Ref. [10].
} 
In light-cone gauge $A^{+}=0$, only the segment at $\xi^{-}= \pm \infty$ contributes and the OAM looks similar to the local manifestly gaguge invariant expression, except

$$
\vec{r} \times \vec{A}(\vec{r}) \longrightarrow \vec{r} \times \vec{A}\left(r^{-}= \pm \infty, \mathbf{r}_{\perp}\right) .
$$

From PT invariance one finds that $\mathscr{L}_{+}^{q}=\mathscr{L}_{-}^{q}$ [5]. In the Bashinsky-Jaffe definition of OAM $\mathscr{L}_{B J}^{q}$ [13], the vector potential in the gauge covariant derivative is replaced by

$$
\frac{\int_{-\infty}^{\infty} d x^{-} A_{\perp}\left(r^{-}, \mathbf{r}_{\perp}\right)}{\int_{-\infty}^{\infty} d x^{-}}=\frac{1}{2}\left[A_{\perp}\left(r^{-}=\infty, \mathbf{r}_{\perp}\right)+A_{\perp}\left(r^{-}=\infty, \mathbf{r}_{\perp}\right)\right]
$$

and is thus equivalent to the light-cone-staple definition

$$
\mathscr{L}_{B J}^{q}=\frac{1}{2}\left(\mathscr{L}_{+}^{q}+\mathscr{L}_{-}^{q}\right)=\mathscr{L}_{+}^{q}=\mathscr{L}_{-}^{q}
$$

Imposing $A^{+}=0$ does not completely fix the gauge as one can still make $r^{-}$-independent gauge transformations. If one fixes this residual gauge invariant by imposing anti-symmetric boundary conditions $A_{\perp}\left(r^{-}=-\infty, \mathbf{r}_{\perp}\right)=-A_{\perp}\left(r^{-}=-\infty, \mathbf{r}_{\perp}\right)$ the vector potential at $r^{-}= \pm \infty$ cancels out in $\mathscr{L}_{+}^{q}+\mathscr{L}_{-}^{q}$ and therefore, with the understanding of anti-symmetric boundary conditions at $r^{-}= \pm \infty$ the Jaffe-Manohar OAM becomes also identical to $\mathscr{L}_{ \pm}^{q}$.

This last observation is crucial for understanding the difference between the Ji vs. JaffeManohar OAM, which in light-cone gauge ${ }^{2}$ involves only the replacement $A_{\perp}^{i}(\vec{r}) \longrightarrow A_{\perp}^{i}\left(r^{-}=\right.$ $\left.\pm \infty, \mathbf{r}_{\perp}\right)$. Using

$$
A_{\perp}^{i}\left(r^{-}=\infty, \mathbf{r}_{\perp}\right)-A_{\perp}^{i}\left(r^{-}, \mathbf{r}_{\perp}\right)=\int_{r^{-}}^{\infty} d z^{-} \partial_{-} A_{\perp}^{i}\left(z^{-}, \vec{r}_{\perp}\right)=\int_{r^{-}}^{\infty} d z^{-} G^{+i}\left(z^{-}, \vec{r}_{\perp}\right)
$$

where $G^{+\perp}=\partial_{-} A^{\perp}$ is the gluon field strength tensor in $A^{+}=0$ gauge. Note that

$$
-\sqrt{2} g G^{+y} \equiv-g G^{0 y}-g G^{z y}=g\left(E^{y}-B^{x}\right)=g(\vec{E}+\vec{v} \times \vec{B})^{y}
$$

yields the $\hat{y}$ component of the color Lorentz force acting on a particle that moves with the velocity of light in the $-\hat{z}$ direction $(\vec{v}=(0,0,-1))$ - which is the direction of the momentum transfer in DIS $[11,14]$. Thus the difference between the Jaffe-Manohar and $\mathrm{Ji}^{3}$ OAMs

$$
\mathscr{L}^{q}-L^{q}=-g \int d^{3} x\left\langle P, S\left|\bar{q}(\vec{x}) \gamma^{+}\left[\vec{x} \times \int_{x^{-}}^{\infty} d r^{-} F^{+\perp}\left(r^{-}, \mathbf{x}_{\perp}\right)\right]^{z} q(\vec{x})\right| P, S\right\rangle /\langle P S \mid P S\rangle
$$

has the semiclassical interpretation of the change in OAM due to the torque from the FSI as the quark leaves the target:[12] while $L^{q}$ represents the local and manifestly gauge invariant OAM of the quark before it has been struck by the $\gamma^{*}, \mathscr{L}^{q}$ represents the gauge invariant OAM after it has left the nucleon and moved to $r^{-}=\infty$.

\section{Intuitive Picture for the Torque from Final State Interactions}

In order to estimate the effect from the final state interactions on the quark OAM we first consider the effect on a positron moving through the magnetic dipole field of an electron, which is polarized in the $+\hat{z}$ direction. This should be the most simple analogy to a proton polarized in the $+\hat{z}$ direction because more quarks are polarized in the same direction as the nucleon spin and the color-electric force between the active quark and the spectators is attractive. As illustrated in Fig.

\footnotetext{
${ }^{2}$ As $L^{q}$ involves a manifestly gauge invariant local operator, it can be evaluated in any gauge.

${ }^{3}$ Here we replaced $\gamma^{0} \rightarrow \gamma^{+}$in $L^{q}$ as discussed in Ref. [15].
} 


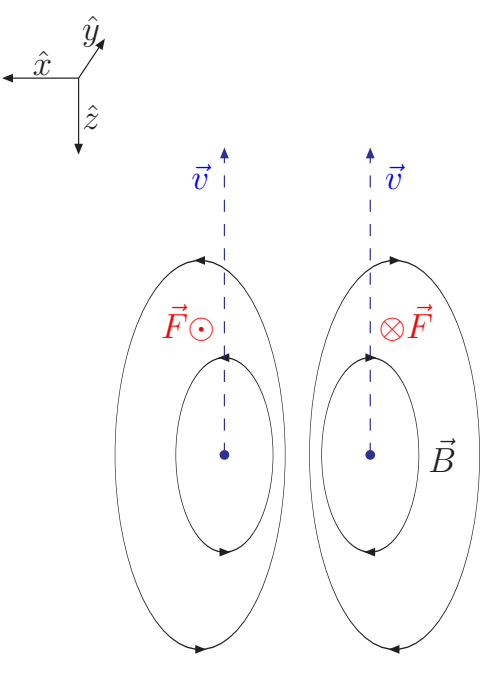

a.) $\otimes \hat{z}$

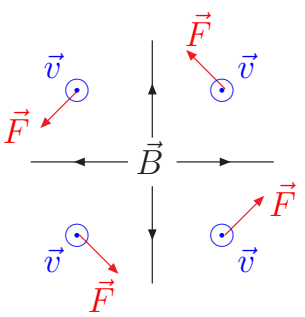

b.)

Figure 2: Illustration of the torque acting on a positron moving in the $-\hat{z}$ direction through a magnetic dipole field caused by the magnetic moment of an electron polarized in the $+\hat{z}$ direction. a.) side view; b.) top view. In this example the $\hat{z}$ component of the torque is negative as the positron leaves the bound state.

2 the magnetic FSI leads to a negative torque. Since the example was chosen such that the signs of polarization and forces are the same as in the nucleon this implies that the color-magnetic torque acting on quarks ejected from the proton are negative as well.

The dipole example also illustrates why the torque vanishes immediately after the absorption of the virtual photon: when comparing the $\perp$ magnetix field (for fixed $\perp$ position) at positions at the 'front' and 'back' side of the nucleon are equal and opposite, and therefore the torque at the original position of the active quark averages to zero

$$
\left\langle P, S\left|\bar{q}(0)\left[x F^{+y}(0)-y F^{+x}(0)\right] q(0)\right| P, S\right\rangle=0 .
$$

Note that this cancellation is a consequence of averaging over all possible initial positions of the active quark. For a specific initial position the initial torque will in general be nonzero. While we used here an intuitive picture to motivate this result, it can also be shown to be a rigorous consequence of PT invariance. 


\section{References}

[1] J. Ashman et al (EMC), 2. A Measurement of the Spin Asymmetry and Determination of the Structure Function $g_{1}$ in Deep Inelastic Muon-Proton Scattering, Phys. Lett. B 206 (1988) 364.

[2] X. Ji, Gauge-Invariant Decomposition of Nucleon Spin, Phys. Rev. Lett. 78 (1997) 610 [hep-ph/9603249].

[3] R.L. Jaffe and A. Manohar, The $g_{1}$ Problem: Fact and Fantasy on the Spin of the Proton, Nucl. Phys. B 337 (1990) 509.

[4] C. Lorcé and B. Pasquini, Quark Wigner Distributions and Orbital Angular Momentum, Phys. Rev. D 84 (2011) 014015 [arXiv: 1106 .0139].

[5] Y. Hatta, Gluon Polarization in the Nucleon Demystified, Phys. Rev. D 84, 041701 (2011) [arXiv:1101.5989]; Notes on the Orbital Angular Momentum of Quarks in the Nucleon, Phys. Lett. B 708 (2012) 186 [arXiv: 1111 .3547].

[6] A.V. Belitsky, X. Ji, and F. Yuan, Quark Imaging in the Proton via Quantum Phase Space Distributions, Phys. Rev. D 69 (2004) 074014 [hep-ph / 0307383 ].

[7] X. Ji, X. Xiong, and F. Yuan, Proton Spin Structure from Measurable Parton Distributions, Phys. Rev. Lett. 109 (2012) 152005 [arXiv: 1202 .2843].

[8] A. Metz,S. Meissner, and M. Schlegel, Nontrivial Relations between GPDs and TMDs, Mod. Phys. Lett. A24 (2009) 2973.

[9] A.V. Belitsky, X. Ji, and F. Yuan, Final State Interactions and Gauge Invariant Parton Distributions, Nucl. Phys. B 656 (2003) 165 [hep-ph / 0307383 ]; D.Boer, P.J.Mulders, and F.Pijlman, Universality of T Odd Effects in Single Spin and Azimuthal Asymmetries, Nucl. Phys. B 667 (2003) $201[$ hep-ph/0303034].

[10] J.C. Collins, What Exactly is a Parton Density?,Acta Phys. Polon. B 34, 3103 (2003) [hep-ph/0304122]; S.M. Aybat et al., The QCD Evolution of the Sivers Function Phys. Rev. D 85, 034043 (2012) [arXiv: 1110 . 6428]; I. Cherednikov, Cusped Light-Like Wilson Loops in Gauge Theories, Phys. Part. Nucl. 44 (2013) 250 [arXiv:1212.4345].

[11] J. Qiu and G. Sterman, Single Transverse Spin Asymmetries, Phys. Rev. Lett. 67 (1991) 2264.

[12] M. Burkardt, Parton Orbital Angular Momentum and Final State Interactions, Phys. Rev. D 88 (2013) 1 [arXiv: 1205.2916].

[13] S. Bashinsky and R.L. Jaffe, Quark and Gluon Orbital Angular Momentum and Spin in Hard Processes, Nucl. Phys. B536 (1998) 303 [hep-ph/9804397].

[14] M. Burkardt, Transverse Force on Quarks in DIS, Phys. Rev. D 88 (2013) 114502 [arXiv:0810.3589].

[15] M. Burkardt and H. BC, Angular Momentum Decomposition for an Electron, Phys. Rev. D 79 (2009) 071501 [arXiv:0812.1605]. 